\title{
The misconceptions about the interpretation of light bulb resistance in specialized school
}

\author{
G. Movsum Oghlu Sharifov \\ Azerbaijan State Pedagogical University, \\ Doctor of Philosophy in Physics, Associate Professor, \\ e-mail: galib_sharifov@mail.ru
}

Received 15 July 2020; accepted 30 August 2020

\begin{abstract}
The paper deals with the interpretation of the resistance of incandescent light bulbs in series and parallel connection. It has been established that the temperature dependence of resistance for these types of lamps always manifests itself in school experiments. Therefore, the basic knowledge related to the temperature dependence of resistance should be taught to the students in a specialized school on the basis of the didactic principles.
\end{abstract}

Keywords: incandescent; light bulb; resistance; series; parallel; specialized school.

DOI: https://doi.org/10.31349/RevMexFisE.18.90

\section{Introduction}

Recent years have witnessed a growing academic interest in teaching methods of the topics related to the electricity in physics subject. A large number of studies in literature has explored the students' difficulties in understanding of electrostatics and electrical circuits as well as strategies that may be useful for them to learn these topics [1-14].

The experimental and theoretical works regarding incandescent light bulb are always of interest to both types of investigator in physics: scientists and pedagogies. Some studies $[15,16]$ attempted to define the physics of incandescent lamps in terms of the technological structure and showed working function.

Some studies investigated the temperature dependency of incandescent lamps [17,18]. Regarding the temperature of the light bulb, Bruce [18] found that the non-linear temperature dependence of the resistivity has a significant effect on a 60-watt lamp. Agrawal et al. [19] using bulbs of 10-1000 W estimated the efficacy of incandescent light bulbs as a function of temperature Darren W. et al. [20]) explored teacher's understanding of power, a potential difference of light bulb in series and parallel circuit. They qualitatively showed that it is difficult for teachers to apply and integrate concepts in physics, closely related topics. In result, they offered the importance of temperature dependence of the bulb resistance in the teaching process of electricity.

Carla [21] comprehensively explored Stefan-Boltzmann radiation law with tungsten filaments of commercial incandescent lamps. It has been described the total dissipated power by the lamp Lubos [22] presented a solution for the problem of the real efficiency of incandescent bulbs when taking into consideration of both light and heat effects of the interior illumination of buildings.

Taking into account the evaporation of half the radius of the filament during the lifetime, Dulli and Jayaram [23] estimated the average evaporation rate. They also noted a possible reason for limiting the life of the incandescent lamp.
According to their explanation, the thin part of the filament is mechanically weak. When the bulb is turned on or off, a sudden thermal change causes mechanical stress that leads to a breakdown of the filament at its thinnest point.

Some authors [24] described the principal electrical and optical characteristics of an incandescent lamp (incandescent light bulbs) in teaching introductory electricity and magnetism.

Recently Jing and Chandralekha [25] have given a comprehensive review on the difficulties of students' reasoning and approach in solving problems about the brightness of non-identical light bulbs in series and parallel circuit. Comparing the results of the work of students and groups of graduate physicists, they have found that these problems are related to non-identical light bulbs.

Despite the theoretical and practical study of the temperature dependences of resistance for the incandescent lamps in the papers mentioned above, there are conflicting opinions among specialized school students on the changes in the resistance value of the lamp during experiments such as series and parallel connection of lamps, as well as its measuring by the devices. This can also be explained by the incorrect organization of the educational process of physics in term of didactic issues. Therefore, in this paper, it was investigated the resistance of incandescent light bulb in series and parallel circuit within school laboratory condition from the didactic principles point of view.

\section{The experiment}

Equipment used in experiments: incandescent light bulbs MH 6.3-03 (E10/13, $6.3 \mathrm{~V}$ and $0.3 \mathrm{~A}$, resistance at $22^{\circ} \mathrm{Cis}$ $3.5 \pm 0.1 \Omega$ ), voltage source rectifier BS-24 DC voltage $0 \sim 10 \pm 0.5 \mathrm{~V}$, DC Ammeter for School Education (Yongjing AMP001, Measuring Range: $-0.2 \mathrm{~A} \sim 0 \sim 0.6 \pm 0.05 \mathrm{~A}$, $-1 \sim 0 \sim 3 \pm 0.05 \mathrm{~A}$ ), DC Analog Voltmeter for Educatio 


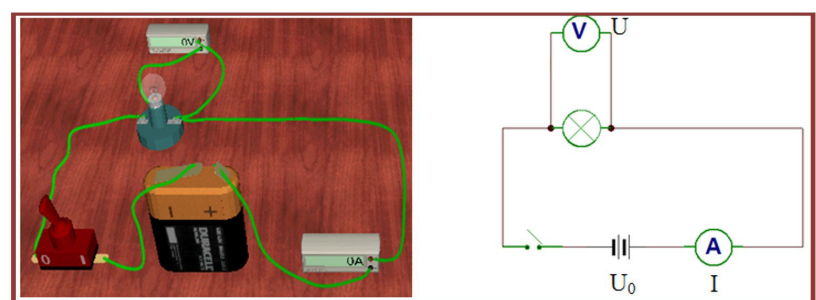

FIGURE 1. Scheme of a lamp circuit (on the right) and its model in the program Edison 5 (on the left).

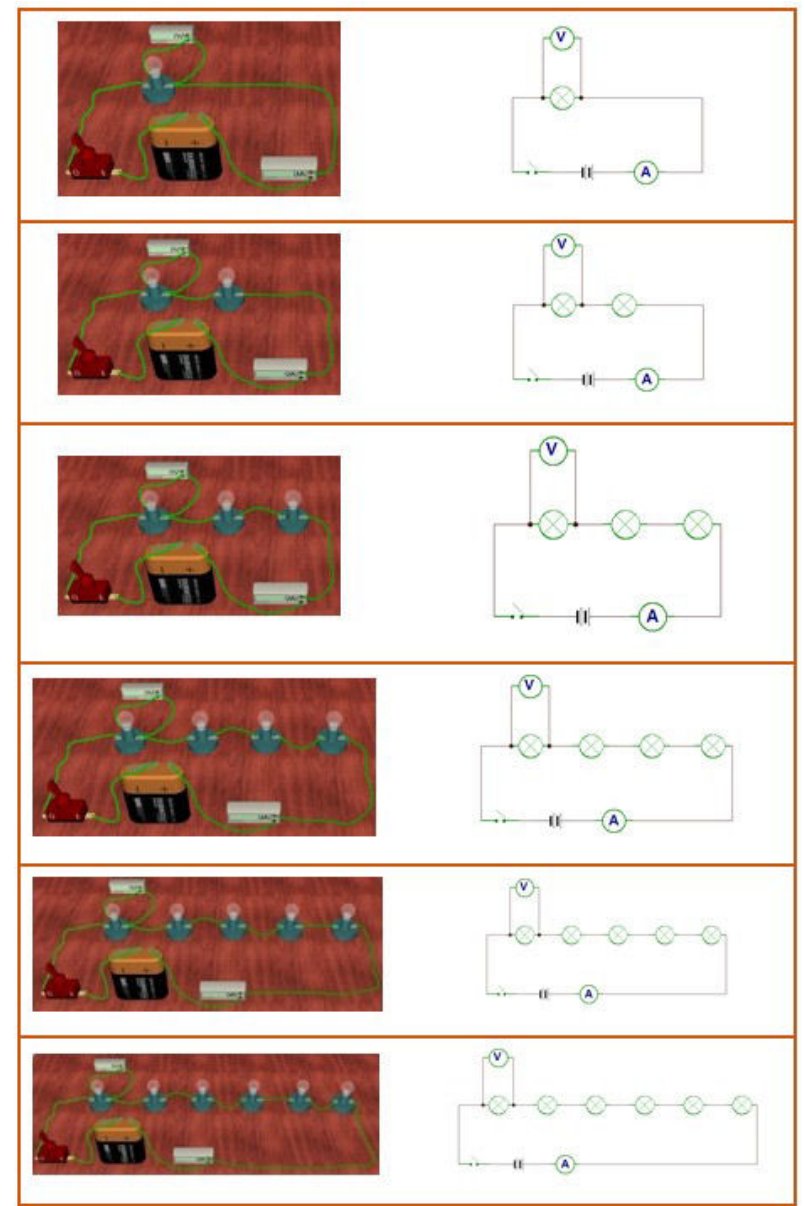

FIGURE 2. Scheme of a series connection of incandescent lamps (on the right) and its model in the program of Edison 5 (on the left).

(Yongjing VLT005 Measuring Ranges: $0 \sim 3 \sim 0.05 \mathrm{~V}$, $0 \sim 15 \pm 0.1 \mathrm{~V}, 0 \sim 300 \pm 2 \mathrm{~V})$, digital Multimeter M3900. Rectifier BS-24 converts an alternating electric current with a frequency of $50 \mathrm{~Hz}$ and a voltage of $220 \mathrm{~V}$ to alternating current with a smoothly regulated voltage from 0 to $30 \mathrm{~V}$ and an electric current up to $10 \mathrm{~A}$, as well as to rectified current with a smoothly regulated voltage from 0 to $24 \mathrm{~V}$ and a current up to $10 \mathrm{~A}$. The maximum power consumed by the rectifier can reach $500 \mathrm{~W}$.

Firstly, the volt-ampere characteristic of the light bulb was registered and modelled using the Edison 5 program (Fig. 1). Afterwards, through formula $(R=U / I)$, the re-

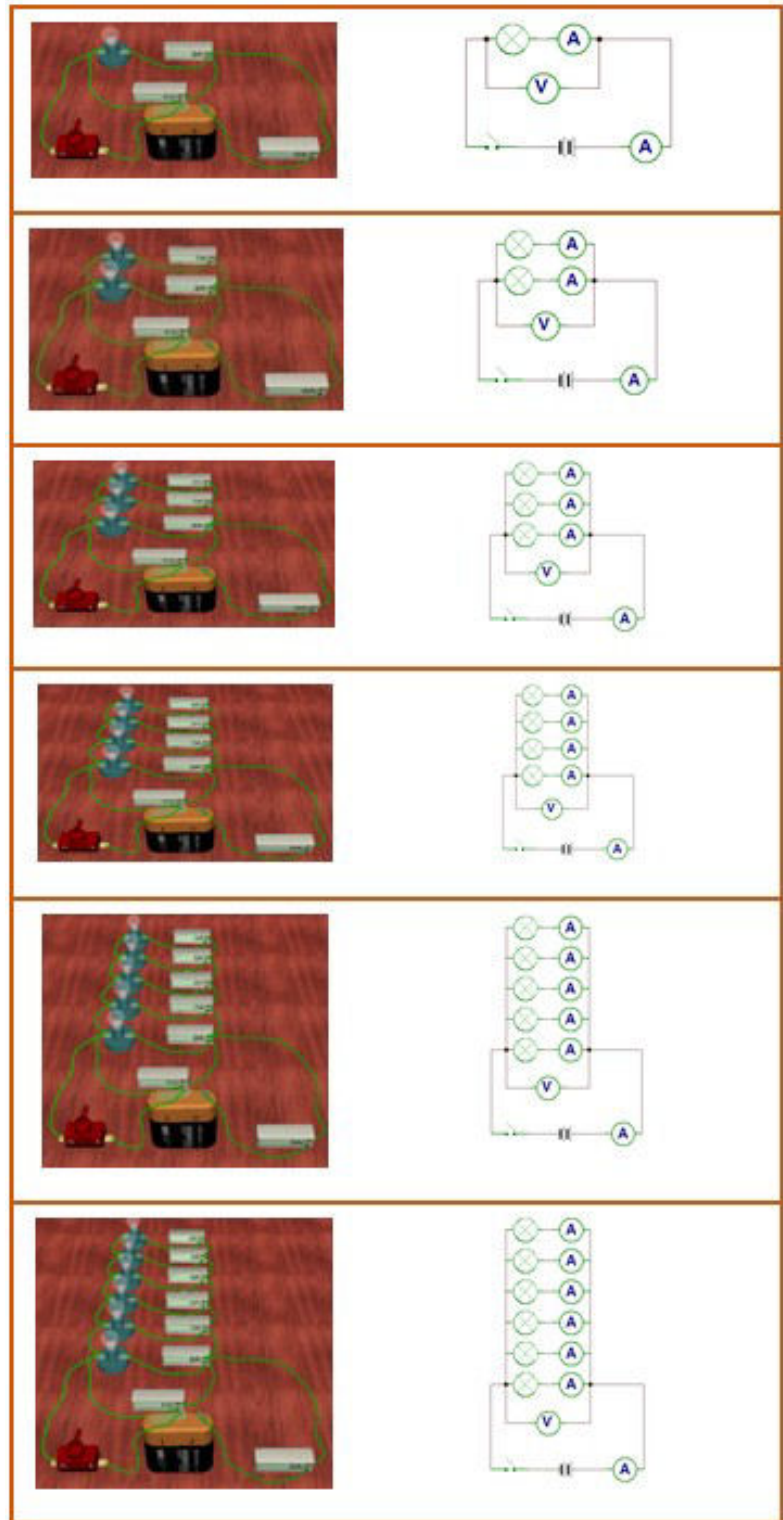

FIGURE 3. Scheme of parallel connection of incandescent lamps (on the right) and its model in the program Edison 5 (on the left).

sistance of the lamp filament is calculated. Furthermore, it has been carried out the experiments about series and parallel connection of the lamps. The readings of the ammeter and voltmeter were recorded (Fig. 2, 3) with sequentially increasing the number of bulbs under the constant value of the current source, i.e. each time when the next lamp connected serially (Fig. 2) and in parallel (Fig. 3) to the circuit, the readings of the voltmeter and the ammeter in the series and parallel circuits are registered.

Furthermore, the results of series and parallel combinations of lamps in the open circuit via a Digital Multimeter M3900 were analyzed. The purpose of the selection of $60 \mathrm{~W}$ bulbs was its being easily accessible for students. However, I have not used these lamps with students in a closed circuit for safety issues. The resistance of the one and two lamps have been consistently registered (Fig. 4). In other words, I 


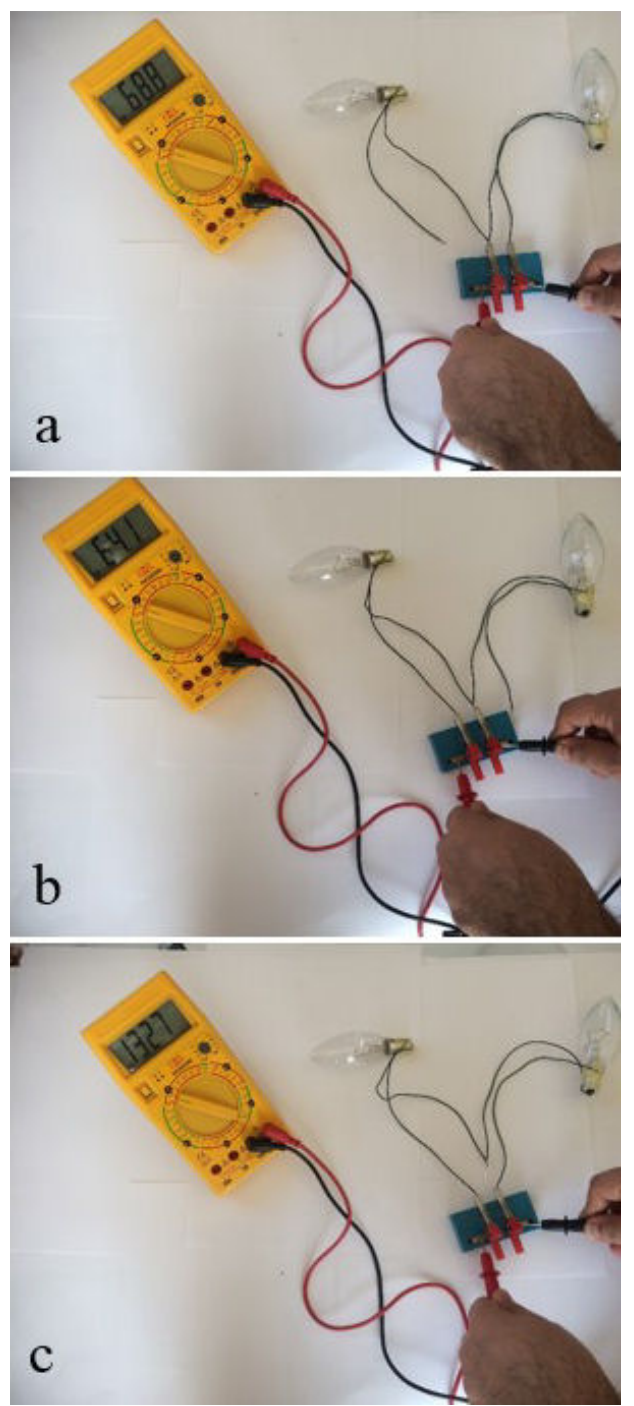

FIGURE 4. Measuring the resistance of lamps with the same power through the Digital Multimeter M3900: separately (a, b), series (c).

I connected light bulbs $(60 \mathrm{~W})$ to a tester and photographed the results of such experiments. In Fig. 4, it is visible that the tester shows a reduced resistance of light bulbs that are switched in series connection.

In Fig. 4, the sum of the resistances measured separately for the light bulbs of $60 \mathrm{~W}: 68.8+64.1=132.9 \Omega$. However, by measuring the resistance in series, the device will "reduce" the reading to $132.7 \Omega$. A similar difference is observed for the lamps of $60 \mathrm{~W}$ and $0.6 \mathrm{~W}$ (Fig. 5 ): $2.1+65.8=67.9 \Omega$. Nevertheless, the device will "reduce" the reading to $67.6 \Omega$.

In result, the formula of $R_{\text {series }}=R_{1}+R_{2}$ shows a decreased value of the resistance of the "cold" filament (filament without electric current running on it) in series connection.

Afterwards, to clarify the "errors" occurred by measuring lamps $60 \mathrm{~W}$ and $0.6 \mathrm{~W}$ with the Multimeter, the resistance of each bulb, measured for 2 hours in an open circuit through the Multimeter. So, the resistance value of a $60 \mathrm{~W}$ lamp was registered $(68.5 \Omega)$ for 2 hours without breaking off the con-
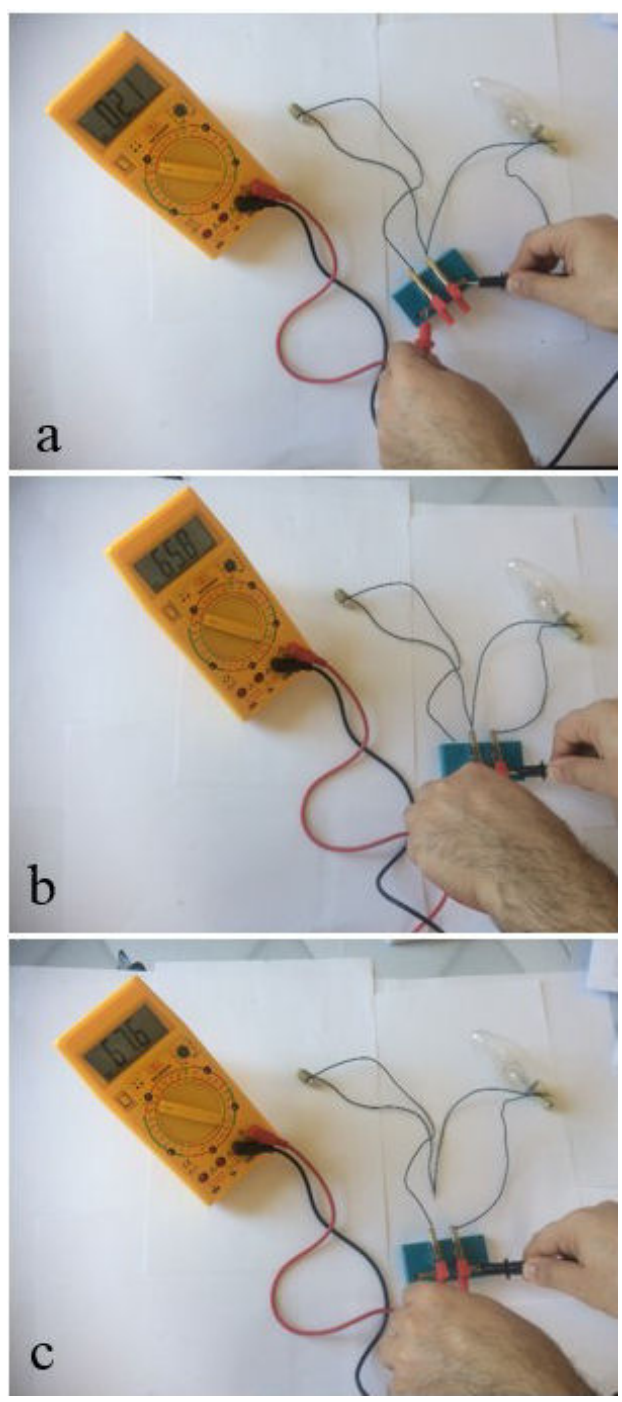

FIGURE 5. Measuring the resistance of the lamps with different power through the Digital Multimeter M3900: a and b separately, c series.

tacts and was not registered any changing. However, the resistance of the lamp $(0.6 \mathrm{~W})$ was $2.1 \Omega$ and at the end of 2 hours, increased up to $2.8 \Omega$. This fact may be explained as follows: if taking into consideration the dissipated power by the bulb and the heat capacity:

$$
\begin{gathered}
Q=I^{2} R_{0} t, \quad Q=m C \Delta T, \\
\Delta R=\alpha R_{0} \Delta T, \quad \Delta R=\frac{\alpha I^{2} R_{0}^{2} t}{\rho V C},
\end{gathered}
$$

where $I$ - electric current, $\Delta R$ - change in temperature, $R_{0}$ initial resistance, $R$ - final resistance, $m$ - mass of conductor, $C$ - specific heat capacity, $\alpha$ - temperature coefficient of resistivity $\Delta T$ - change in temperature, $V$ - the volume of the conductor, $\rho$ - the density of conductor, $t$ - time. Thus, it is expected that the resistance change as the time increases. These observed facts must be taken into account, and it should be informed to students during experiments regarding the connection of lamps. 


\begin{tabular}{lccccc}
\hline \hline TABLE I. "Cold" and "hot" resistances of lamps with different power. & & & & \\
\hline Power of lamps, W & 25 & 40 & 60 & 75 & 100 \\
"cold" resistance of lamp filament, $\Omega$ & 150 & $92-99$ & $63-66$ & $42-48$ & $36-42$ \\
"hot" resistance of lamp filament, $\Omega$ & 1936 & 1210 & 807 & 645 & 484 \\
$R_{\text {hot }} / R_{\text {cold }}$ & 12 & 12 & 13 & 13 & 12 \\
\hline
\end{tabular}
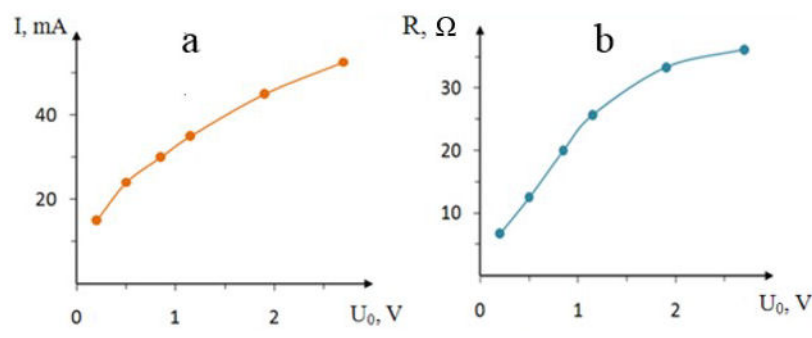

FIGURE 6. The dependence of the electrical current (a) and resistance of lamp (6.3 V and $0.3 \mathrm{~A})(\mathrm{b})$ from the voltage source.

At last, in order to make a comparison between those obtained facts, it has experimented with the parallel connection of lamps. The readings of the ammeters, voltmeter, and ammeter in the circuit were registered by connecting each lamp continuously in a parallel circuit under the constant value of the voltage source (Fig. 3).

\section{Results and discussion}

From the experiments, the graph of the volt-ampere characteristics of a lamp was presented in Fig. 6a. Then, through precise calculations

$$
I=\frac{U_{0}}{R}, \quad R=\frac{U_{0}}{I}
$$

and taking into account the internal resistance of the Rectifier BS-24 $(\approx 8 \Omega)$, the curves showing the dependencies of electric current $(I)$ and resistance of the lamp $(R)$ from the voltage source $\left(U_{0}\right)$, are presented in Fig. 6.

Figure 6 shows that the non-linear dependences $I\left(U_{0}\right)$ and $R\left(U_{0}\right)$. But the literature indicates that for a resistor, these dependencies must be linear. After showing the student the different results between filament and resistor, this "phenomenon" can be explained to them as follows: the incandescent filament of the lamp is tungsten, and as the temperature of the metal increases, its resistance also increases, i.e. with increasing the voltage source, the electric current in the circuit increases. It leads to a rise in the amount of heat released from the filament following the Joule-Lenz law. However, the theoretical part of this physical phenomenon is comprehensively described in some papers [18-20]. But there are no didactic aspects of this phenomenon in the literature. Therefore, students should be encouraged to explain in detail why it is necessary to choose metal like tungsten for the incandescent lamp, and why the diameter of its cross-section is 40 microns, and the length is $50 \mathrm{~cm}$ as well as by which way its length in a lamp is created like an about $2 \mathrm{~cm}$. Students can systematically conclude research in the laboratory: the rising electric current through the incandescent filament with increasing the value of voltage source (U0) (Fig. 6a) begins to heat it, its resistance is increased (Fig. 6b). This increase in its turn prevents a linear increase in electric current with growing resistance of the lamp. Besides, in order to show the difference between "cold" and "hot" resistance of the light bulbs. I measured several the "cold" and "warm" resistance of incandescent lamps under AC voltage source of $220 \mathrm{~V}$ (Table I). As shown in Table I, the resistance of the lamps in the "cold" condition is minimal; however, the passing electric current through the filament increases its resistance by 12-13 times.

An ohmmeter measures the resistance of the lamp used in our experiment, and the "cold" resistance is $3.5 \Omega$. However, according to the nominal values on the lamp $(6.3 \mathrm{~V}$ and 0.3 a), the resistance of this lamp $(6.3 \mathrm{~V} / 0.3 \mathrm{~A}=21 \Omega)$ is $21 \Omega$. The "hot" resistance of the lamp, which we use in the experiment, is six times greater than the "cold" one. Therefore, in investigations related to the resistance of lamps, students should be explained the reason of the difference between the resistance they received from the nominal readings, and the "cold" resistance measured by an ohmmeter, and its effect on electrical circuits.

It is shown from the dependencies obtained from the experiments carried out with one lamp (Fig. 7a), i.e. it was presented the dependencies resistance and electric current running through it from the voltage drop of the lamp, registered by the voltmeter. As a result, it is observed a non-linearity in these dependencies.

The non-linear increase of voltage drop may be explained by two factors ( $\left.U=I R_{\text {lamp }}\right)$. The first is the electric current $(I)$ and second is the resistance of the lamp $\left(R_{\text {lamp }}\right)$. We know that when the value of voltage source increases, the electrical
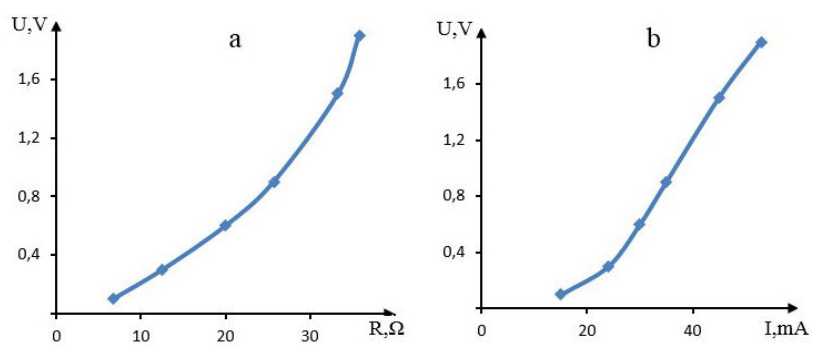

FIGURE 7. The dependence of voltage drop from the lamp resistance (a) and the electric current passing through the lamp (b) in one lamp circuit. 

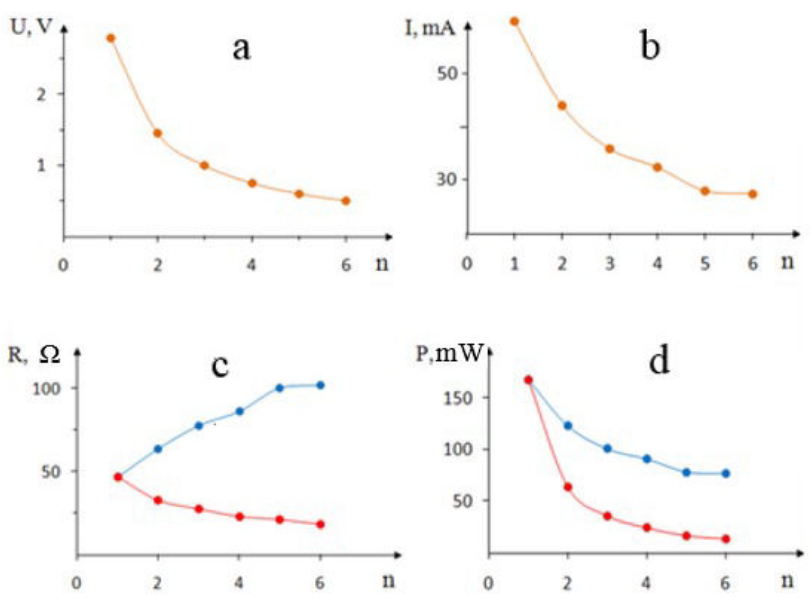

FIGURE 8 . In the series connection of lamps depends on the number of lamps from voltage drop (a) and electric current (b), the total resistance (c, blue), the resistance of the bulb (c, red), the total power (d, blue), the power of one lamp (c, red).

current increases (Fig. 7b), but at the same time the temperature also increases, and this fact in its turn, leads to rising of the resistance. Here the students may face a problem. Because, when the resistance of the lamp filament increases $(R \uparrow)$, the current passing through it decreases $(I \downarrow)$. As a result, the value of the voltmeter is expected to remain constant according to the equation of $U=I R$.

Nevertheless, this constant was not observed. This can be explained to students as follows: as the value of voltage source increases, the temperature of filament increases with the increasing electric current passing through the lamp. This also leads to an increase in resistance and a decrease in electrical current. This decrease prevents a linear increase in the electric current (Fig. 6a). However, if you take a resistor instead of a lamp, the resistance increase will not be observed. In this regard, the physics teacher may also compare the ammeter and voltmeter readings in circuits with the resistor and lamp. It can also give students an in-depth knowledge of lamp resistance calculation.

After analyzing the readings of the ammeter and voltmeter in the circuit with the lamp, students need to be aware of the difference between features of a series and parallel combination of lamps. Therefore, after studying the characteristics of an incandescent bulb, students may show great interest in the investigations of its series connection. For this purpose, the ammeter and voltmeter readings in the circuit are recorded by series connecting the lamps in turs without changing the value of the voltage source $\left(U_{0}=2.8 \mathrm{~V}\right)$ (Fig. 2). The certain formulas are used to calculate the resistance of each bulb $\left(R_{\text {lam }}\right)$, voltage drop $(U)$ and its power $\left(P_{\text {lam }}\right)$, as well as total circuit resistance $\left(R_{t}\right)$ and total power $\left(P_{t}\right)$.

$$
R_{\mathrm{lam}}=\frac{U}{I}, \quad R_{t}=\frac{U_{0}}{I}, \quad P_{\mathrm{lam}}=U I, \quad P_{t}=U_{0} I .
$$

The graph (Fig. 8c, blue) shows that as the number of lamps connected in series to the circuit increases, the total resistance also increases. In other words, despite the decreasing the "hot" resistance of each series-connected lamps, the total resistance of them rises.

The curves show that as the number of lamps connected in series increases, the voltage at the ends of the lamp drops (Fig. 8a) and the electric current in the circuit decreases (Fig.8b). Because when the number of lamps increases, the total resistance increases (Fig. 8c, blue) and it results in a reduced electric current. But the cost of reducing the electric current, depending on the resistance, is less than the cost of its reduction by increasing the number of lamps. This also leads to a decrease in the voltmeter value $(U=I R)$ (Fig. 8a). Besides, the electrical resistance of each bulb is reduced (Fig. 8c, red) and the power (Fig. 8d, red) decreased too. This fact also means that the brightness of the lamps is reduced. This change in lamps can be explained as follows: the number of series-connected lamps circuit $n \uparrow \Rightarrow$ total resistance in the circuit $R_{t} \uparrow \Rightarrow$ the current in the circuit $I \downarrow \Rightarrow$ quantity of heat that passes through the lamp $Q \downarrow \Rightarrow$ temperature of tungsten wire $T \downarrow \Rightarrow$ resistance of tungsten wire $R \downarrow \Rightarrow$ the resistance of the lamp decreases.

One interesting fact related to the series combination of lamps would be more appropriate to show to the students. So, when a certain number of lamps are connected in series in a closed-loop, the final resistance is higher than the total resistance in an open loop. It is because the resistance of incandescent lamps in a closed loop is higher than in the opened loop. In this regard, the experience conducted with $60 \mathrm{~W}$ bulbs will help students to acquire more in-depth scientific knowledge about the serial connection of lamps in an open circuit (Fig. 4, 5).

As we know from the general course of physics any, even the smallest electric current heating the wiring will have a thermal effect on it. By measuring the resistance of a light bulb with a multimeter, we pass a current through it. The electric current from the Multimeter is smallest, but it exists. Therefore, by measuring the resistance of the filament, we heat it; as a result, the value of the particular parameter changes. Roughly speaking, the Multimeter is also "lying". The Multimeter does not show the real value of the filament resistance. In order to make sure of this circumstance, you can do a simple experiment.

You can use the same Multimeter to select two bulbs with the same "cold" resistance, and measure the resistance of two bulbs: first separately, and then connected in series. Repeated measurements show that the sum of the resistances measured separately does not match the total resistance of the series connection. In other words, I measure the resistances of light bulbs individually. Then I measure the resistance of the serial connection. Even though the accuracy of Digital Multimeter M3900 is in range $200 \Omega \pm 0.1 \%$ of a reading digit, I have consistently observed that the sum of resistances measured "singly" is higher than the total resistance of light bulbs included in series (Fig. 4, 5). It is because the series resistance of the two coils decreased the current from the Multimeter, and the filaments heat up less. In a series circuit, if the volt- 

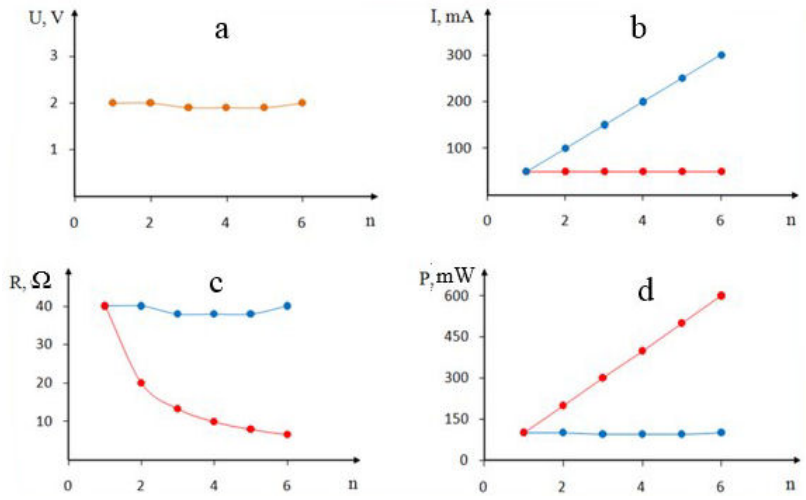

FIGURE 9. In a parallel combination of lamps the dependency of the voltage drop of the bulb (a) and the electric current passing through the lamp (b, red), the total electric current passing through the cycle (b, blue), the total resistance (c, red), the resistance of one lamp (c, blue), the total power (d, red), the power of one lamp (d, blue) from the number of lamps.

age applied is constant, the current is the same for all elements [26]. Moreover, when we measure the resistance of the light bulbs separately, the current appeared by the Multimeter became higher and as a result, the small increase in the temperature of the filament is accompanied with the growth in its resistance. This fact manifests itself in measuring device, i.e. the readings of the Multimeter increase accordingly due to the heating process of filament during the measurement.

Previously, it was impossible to detect this difference using the analogue ohmmeter. Now in any house, there is a digital Multimeter, and anyone can do this simple experiment. The difference in resistance is small, but the difference is noticeable.

When studying some of the results obtained from the serial connection of incandescent lamps, it is appropriate to conduct experiments on its parallel connection. For this purpose, the electrical circuit was assembled (Fig. 3). Then the readings of ammeters and voltmeters in the circuit are registered without changing the voltage source $\left(U_{0}=2 \mathrm{~V}\right)$ with a simultaneous connection of lamps $(6.3 \mathrm{~V}$ and $0.3 \mathrm{~A})$ to the electrical circuit (Fig. 9).

When the next lamp sequently is connected parallel in each branch, the ammeter is also connected in the same branch. In other words, as the number of lamps increases, so the number of ammeters in the circuit increase too. Based on data obtained from experience on parallel connection, the resistance of each lamp $\left(R_{\mathrm{lam}}\right)$, the total resistance $\left(R_{t}\right)$, the power released in the circuit $(P)$, the current passing through the lamp $\left(I_{\text {lam }}\right)$, the total current passing through the cycle $\left(I_{t}\right)$, the voltage drop $(U)$, the total power $\left(P_{t}\right)$ and the power of the lamp $\left(P_{\text {lamp }}\right)$ were analyzed among the students.

The pictures show that as the number of lamps connected in parallel to the circuit increases, the voltage drop at the ends of the lamps (Fig. 9a) and the value of ammeter (Fig. 9b, red) do not change. As a result of the following equations, it became known that the lamp resistance (Fig. 9c, blue) and its power (Fig. 9d, blue) do not change.

$$
R_{\mathrm{lamp}}=\frac{U}{I_{\mathrm{lam}}}, \quad P_{\mathrm{lam}}=U I_{\mathrm{lam}} .
$$

This fact may be explained like this: increasing the number of parallel-connected lamps to the circuit leads to rising in the total current passing through the cycle $\left(I_{t}\right)$. However, the current passing through each lamp $\left(I_{\text {lam }}\right)$ becomes the same due to the dividing the total electric current into the number of branches. Besides, with the increasing the number of parallel-connected lamps, the total resistance decreases (Fig. 9c, red), however the electric current increases (Fig. 9b, blue).

Being paid attention to curves in Fig. 8a and in Fig. 9b (blue), one can conclude that the curve in Fig. 9b (blue) is in agreement with circuits in parallel. Because unchanging of the resistance for one lamp (Fig. 9c, blue) leads to an increase of the total electric current passing through the cycle (Fig. 9b, blue). However, in the case of Fig. 8a, the behaviour of the curve is not a straight line as is expected for circuits in series. On the one hand, the non-linear in Fig. 8a could be due to the decreasing the resistance of each lamp with decreasing temperature of tungsten that caused by the increasing the series-connected lights. On the other hand, despite resistance generally increases with temperature, however for only small temperature changes the resistivity varies linearly with temperature: $\rho=\rho_{0}(1+\alpha \Delta T)$, where $\alpha$ is the temperature coefficient of resistivity. Nevertheless, in our case, an addition of the second series-connected bulb to the circuit resulted in decreasing of resistance for each lamp from 38.8 to $27.9 \Omega$. It means that using the formula of $R=R_{0}(1+\alpha \Delta T)$, with $\alpha$ (tungsten $)=4.5 \times 10^{-3} /{ }^{\circ} \mathrm{C}$, the $\mathrm{DT}$ is around $-62^{\circ} \mathrm{C}$. Therefore, it does not change linearly. This fact manifests itself in the voltage drop of one bulb. In other words, taking into consideration the formula of voltage drop $\left(U=I R_{\text {lamp }}\right)$ for one lamp, then non-linear decrease of voltage drop can be affected by two factors: not the straight rise of total resistance with increasing of series-connected lamps (Fig. 8c, blue) and not straight reduce of the electric current that passes through the bulb (Fig. 8b).

As can be seen from those as mentioned above, the specific contradictory facts (Fig. 8c, red) are revealed in the series-connected electric circuits of lamps, as well as in the measurement of the lamp resistance with a digital multimeter. The physics teacher should systematically explain to the students the reasons for these facts from a didactic point of view and continuously keeps the events related to the temperature dependence of the lamp tungsten filaments on the temperature in the centre of attention, as well as the choice of the correct teaching methods in the process of their teaching process.

\section{Conclusion}

The above-reviewed and -studied experiments in the specialized school laboratory showed that students due to highly cre- 
ative, searchable abilities faced different hardships and misconceptions while exploring the resistance of light bulbs. For this reason, the physics teacher should give detailed and more in-depth knowledge to those students about these misunder- standings. Otherwise, the students will observe multiple "device errors" in physics laboratory works regarding the resistance of the light bulbs.
1. B. Eylon and U. Ganiel, Macro-micro relationships: the missing link between electrostatics and electrodynamics in students' reasoning, Int. J. Sci. Educ. 12 (1990) 79.

2. L. McDermott, and P. Shaffer, Research as a guide for curriculum development: an example from introductory electricity: I. Investigation of student understanding, Am. J. Phys. 60 (1992) 994.

3. L. McDermott, and P. Shaffer, Research as a guide for curriculum development: an example from introductory electricity: II. Design of instructional strategies, Am. J. Phys. 60 (1992) 1003.

4. B. Thacker, U. Ganiel, and D. Boys, Macroscopic phenomena and microscopic processes: student understanding of transients in direct current electric circuits, Am. J. Phys. Suppl. 67 (1999) S2.

5. F. Itza-Ortiz, S. Rebello, and D. Zollman, Students' models of Newton's second law in mechanics and electromagnetism, Eur. J. Phys. 25 (2004) 81.

6. Z. Isvan, and C. Singh, Improving student understanding of Coulomb's law and Gauss's law, AIP Conf. Proc. 883 (2007) 181.

7. J. Li, and C. Singh, Students' difficulties with equations involving circuit elements, AIP Conf. Proc. 1413 (2012) 243.

8. J. Bilak, and C. Singh, Improving students' conceptual understanding of conductors and insulators, AIP Conf. Proc. 951 (2007) 49 .

9. C. Guruswamy, M. Somers, and R. Hussey, Students' understanding of transfer of charge between conductors,

10. J. Guisasola, J. Zubimendi, J. Almudi, and M. Ceberio, The evolution of the concept of capacitance throughout the development of the electric theory and the understanding of its meaning by university students, Sci. Educ. 11 (2002) 247.

11. J. Guisasola, J. Zubimendi, and K. Zuza, How much have students learned? Research-based teaching on electrical capacitance, Phys. Rev. Spec. Top. Phys. Educ. Res. 6 (2010) 020102.

12. P. Engelhardt, and R. Beichner, Student understanding of direct current resistive electrical circuits, Am. J. Phys. 72 (2004) 98 .
13. D. Maloney, T. O'Kuma, C. Hieggelke, and A. Van Heuvelen, Surveying students' conceptual knowledge of electricity and magnetism, Am. J. Phys. Suppl. 69 (2001) 12.

14. C. Efthimiou, D. Maronde, T. McGreevy, E. del Barco, and S. McCole, Implementing elements of The Physics Suite at a large metropolitan research university, Phys. Educ. 46 (2011) 421.

15. D. MacIsaac, G. Kanner, and G. Anderson, Basic physics of the incandescent lamp (lightbulb), Phys. Teach. 37 (1999) 520.

16. H. S. Leff, Illuminating physics with light bulbs, Phys. Teach. 28 (1990) 30.

17. V. Zanetti, Temperature of incandescent lamps, Am. J. Phys. 53 (1985) 546.

18. D. Bruce, The temperature of a lightbulb filament, Phys. Teach. 40 (2002) 101.

19. D. Agrawal, H. Leff, and V. Menon, Efficiency and efficacy of incandescent lamps, Am. J. Phys. 64 (1996) 649.

20. W. Darren, L. Paul, and F. See, Which bulb is brighter? It depends on connection! Strategies for illuminating electrical concepts using light bulbs, Phys. Educ. 52 (2017) 10.

21. M. Carla, Stefan-Boltzmann law for the tungsten filament of a light bulb: revisiting the experiment, Am. J. Phys. 81 (2013) 512.

22. P. Luboš, What is the real efficiency of bulbs?, Physics Education. 47 (2012) 551.

23. D. Agrawal, and V. Jayaram, Lifetime and temperature of incandescent lamps, Phys. Educ. 33 (1998) 55.

24. M Dan, K. Gary, and A. Graydon, Basic physics of the incandescent lamp (Lightbulb), Phys. Teach. 37 (1999) 520.

25. L. Jing, and S. Chandralekha, Students' common difficulties and approaches while solving conceptual problems with nonidentical light bulbs in series and parallel, Eur. J. Phys. 37 (2016) 065708

26. R. Boylestad, Introductory Circuit Analysis, Global Edition. (Pearson Education UK 2015). 